\title{
الطبيعة القانونية لعقد حفظ الحق في بيع العقار المقرر بناؤه
}

$$
\begin{aligned}
& \text { كنزة مخناش } \\
& \text { كلية الحقوق } \\
& \text { جامعة الإخوة منتوري } \\
& \text { قسنطينة } 1 \text { (الجز ائر) }
\end{aligned}
$$

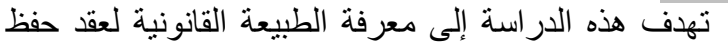

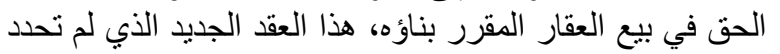

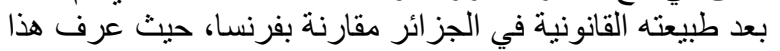

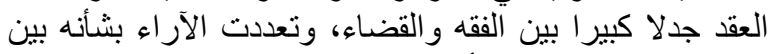

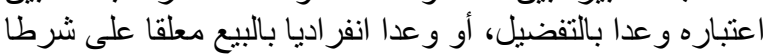
واقفا، أو اعتباره عقد ذو طبيعة مزدوجة ألفة أو عقد ذو طبيعة

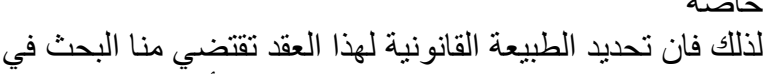

التحليل الفقهي و القضائي الفرنسي، لمعرفة لهيد أي طبيعة قانونية ينتمي إليها هذا العقد.

و هذا ما سوف نحاول الإجابة عنه من خلال هذه الدراسة.

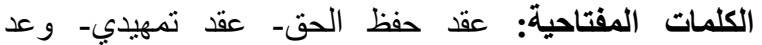

بالتفضيل- وعد انفرادي بالبيع- عقد ذو طبيعة خاصة- عقد ذو

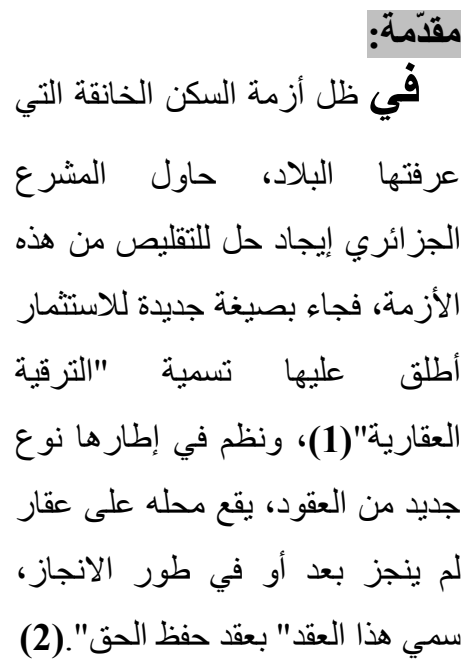

\begin{abstract}
:
This study aims to define the legal nature of the reservation contract in the sale of building to be constructed, this new contract, which has not yet determined the legal nature in Algeria compared to France, where this contract was controversial, There were several opinions on this subject between considering it as a pact of preference, or a unilateral promise of sale under suspensive condition, or be regarded as a double nature contract, or a sui generis contract.

Therefore, the determination of the legal nature of the contract requires us to research in the analysis of doctrinal and French jurisprudential, to know the nature belongs to this contract.

The key- words : réservation contract- préliminary contaract - pact of preference- unilatéral promise of salesui generis contract- double nature contract.
\end{abstract}


هذا الأخير الذي استوحاه المشرع الجزائري من نظيره الفرنسي، والمعروف في فرنسا باسم العقد

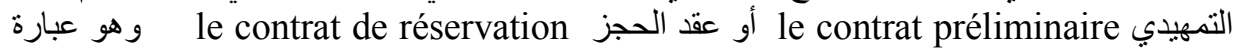
عن عقد تمهيدي، يمهد لإبرام عقد البيع النهائي الناقل للملكية العقارية، يلتزم بمقتضاه المرقي العقارئ العقاري

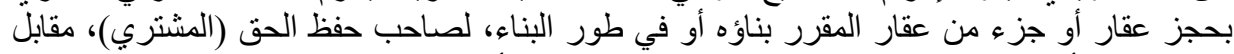

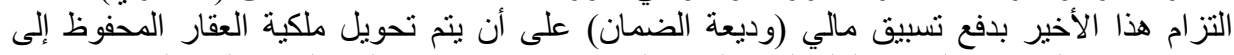

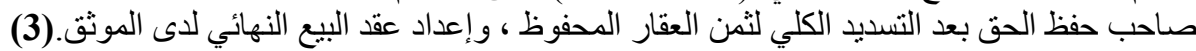

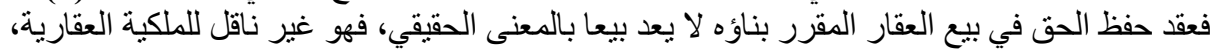

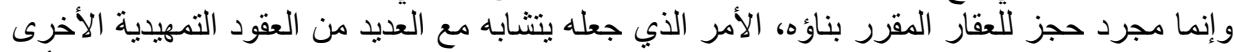

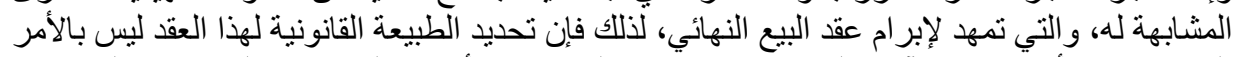

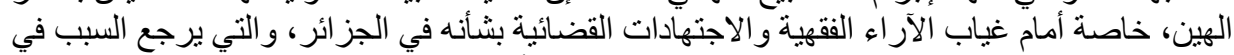

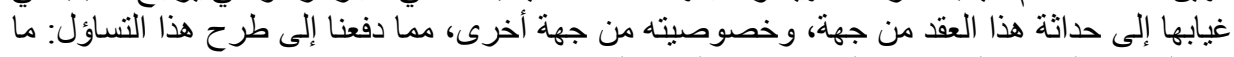

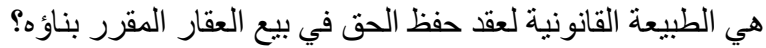

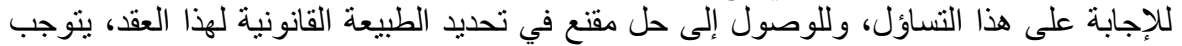

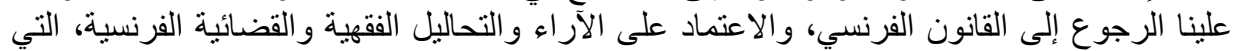

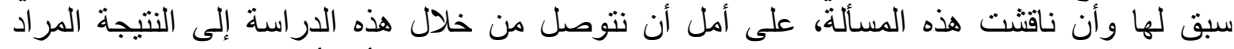

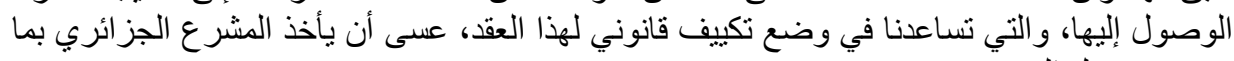
سوف نتوصل إليه. - الئ

ومر اعاة لذللك، سوف نقتصر دراسة الطبيعة القانونية لعقد حفظ الحق في بيع العقار المقرر بناؤه،

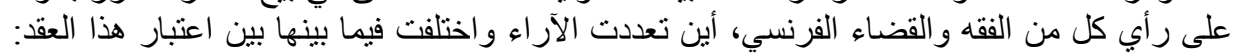

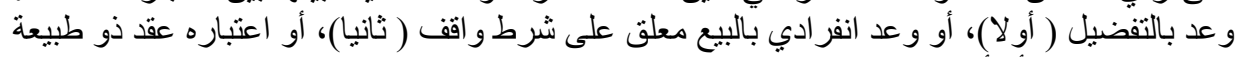

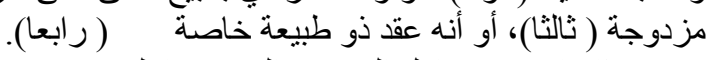

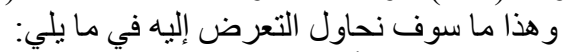

أولا : الاتجاه القائل بأن عقد حفظ الحق وعد بالتفضيل

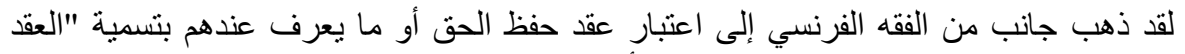

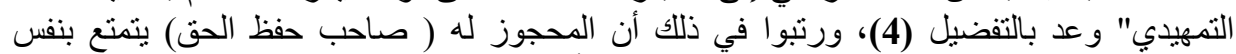

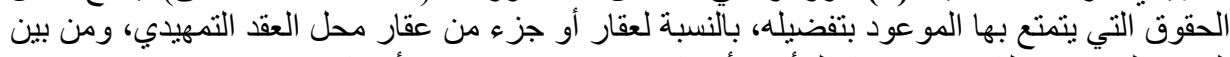

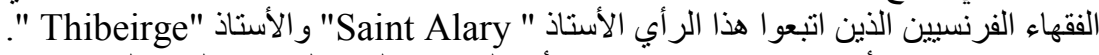

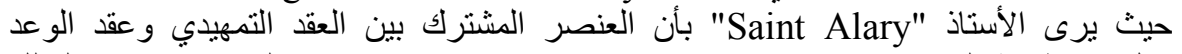

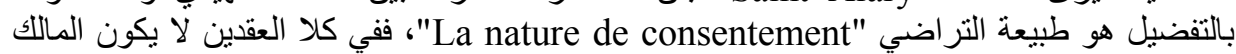

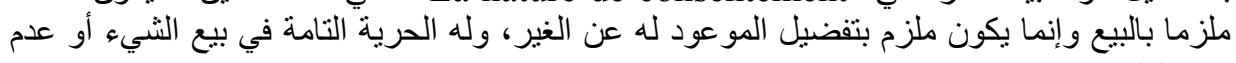

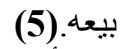

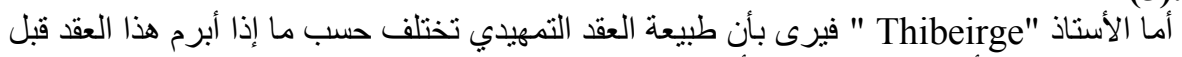

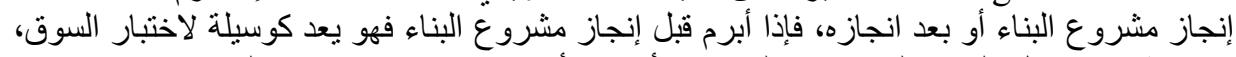

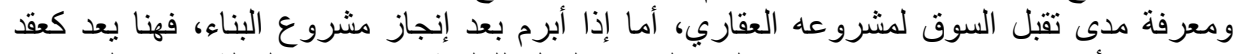

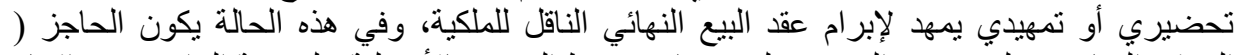

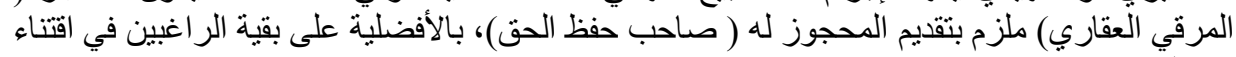

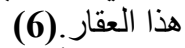

غير أن هذا التحليل أستبعد من قبل الفقه والقضاء، كون هذا العقد لا يتفق مع فكرة الو عد بالتفضيل وذلك في عدة أوجه: 
فمن حيث تقدير الثمن نجد أنه في العقد التمهيدي، ينم الاتفاق على الثمن من قبل أطراف العقد،

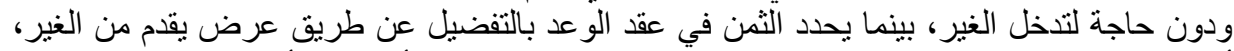

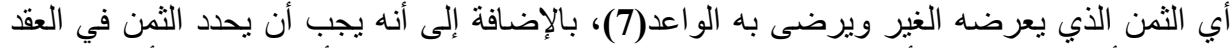

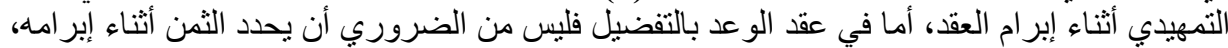

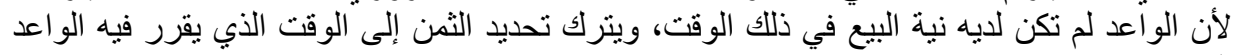

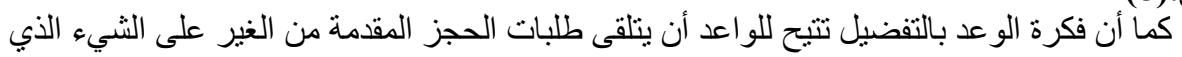

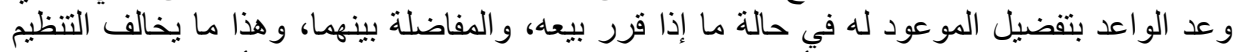

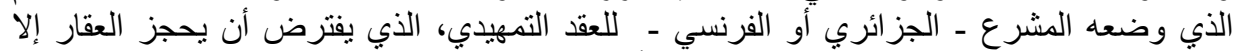

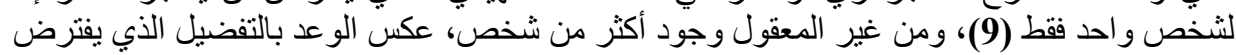

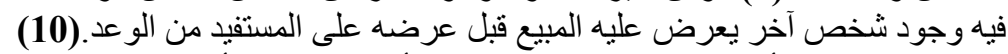

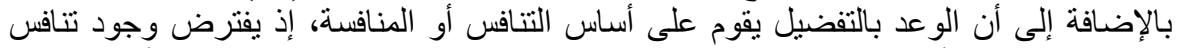

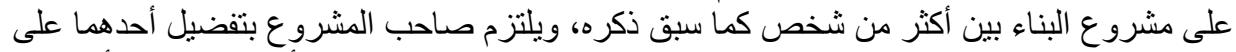

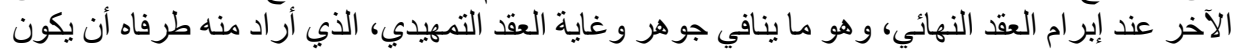

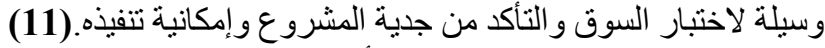

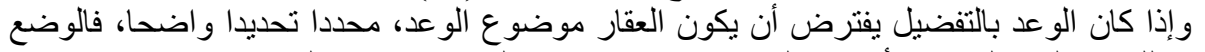

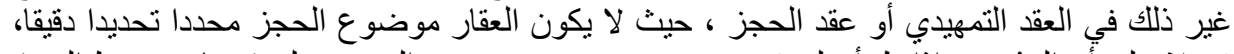

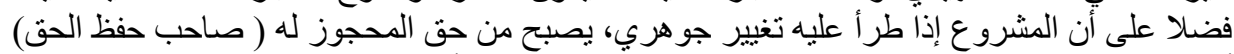

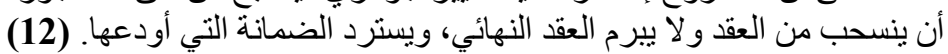

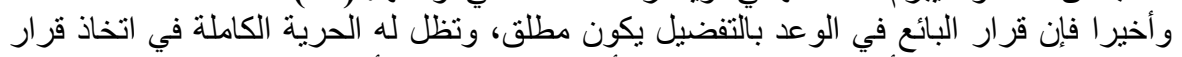

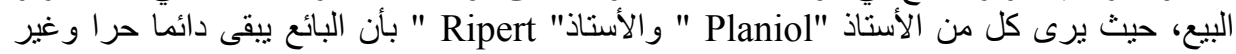

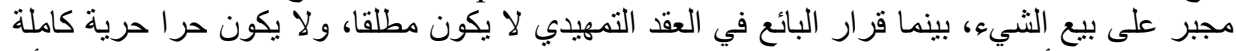

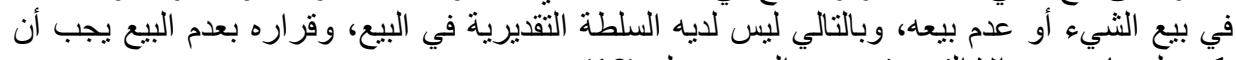

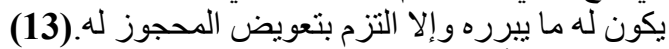

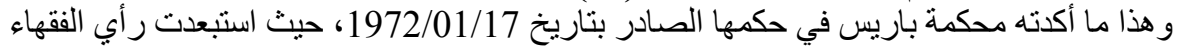

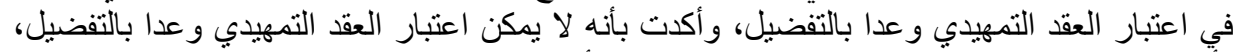

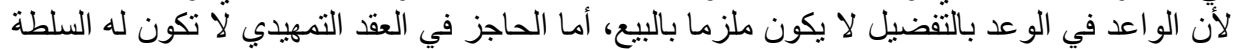

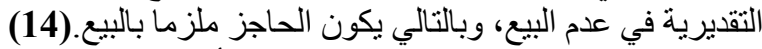

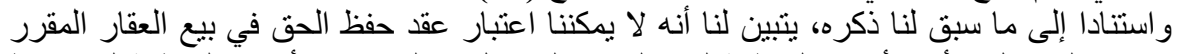

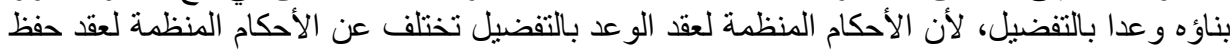
الحق، وبالتالي لا يمكنا تطبيقها عليه، وعليه فإن هذا العقد العقد يعد صورة جديدة من اتفاقات ما قبل التعاقد مستقلة بذاتها عن الوعد بالتفضيل.

\section{ثانيا: الاتجاه القائل بأن عقد حفظ الحق وعد انفرادي بالبيع معلق على شرطو اقف.}

لقد ذهب جانب أخر من الفقه والقضاء الفرنسي إلى اعتبار العقد التمهيدي و عدا انفر اديا بالبيع معلق على شرطو اقف، هو تنفيذ مشرو عن البن البناء.

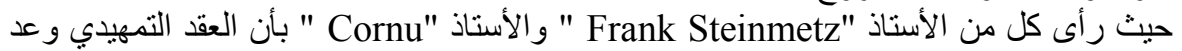

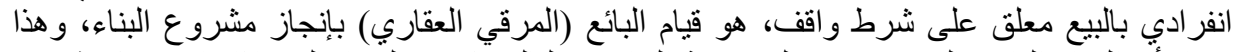

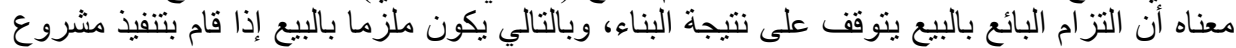

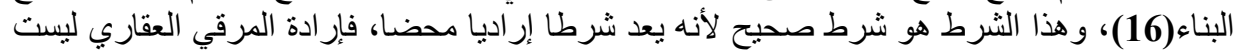


مطلقة بل مقيدة بالظروف الخارجية، وخاصة الظروف الاقتصادية التي تسمح له بتحقيق مشروع

البناء.(17)

فحسب هذا الر أي فإن البائع يلتزم بالبيع، و لا يلتزم المشتري بالثر اء رغم إيداع هذا الأخير لوديعة

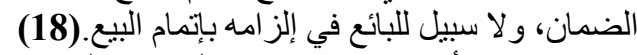

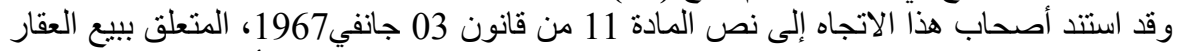

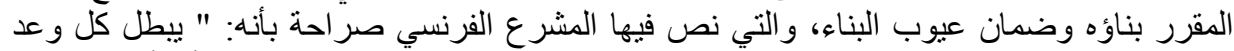

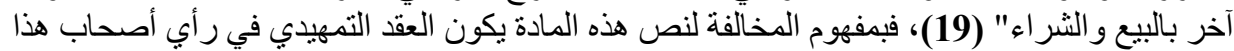

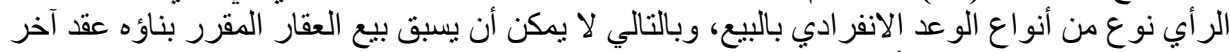
غير العقد التمهيدي المنظم بأحكام خاصنة.

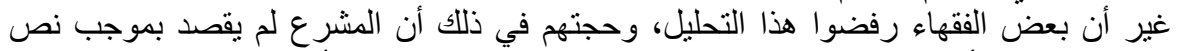

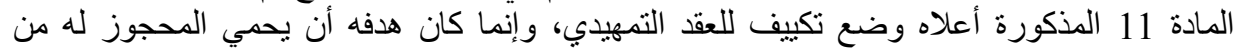

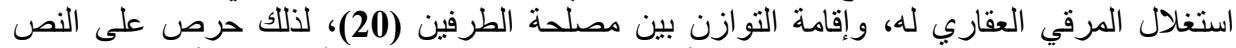

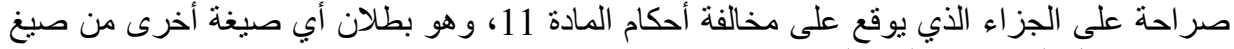
اتفاقات ما قبل التعاقد عدا العقد التمهيدي.

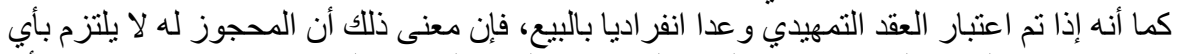

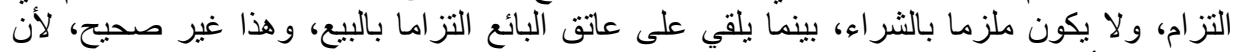

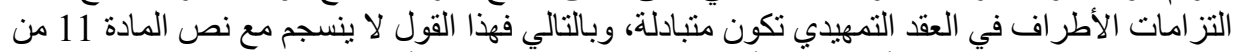

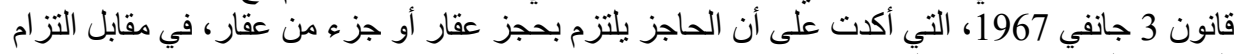

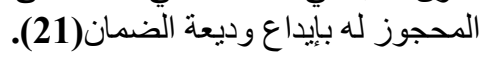

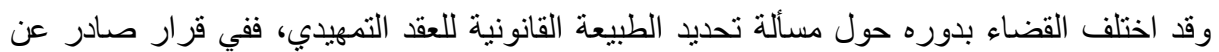

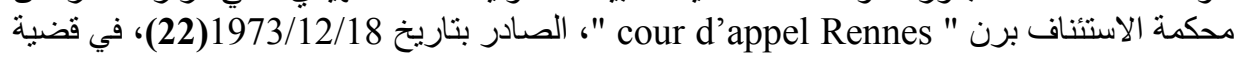

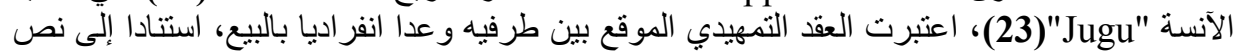

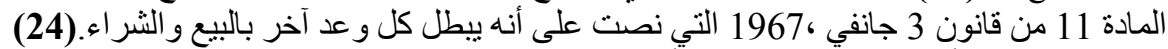

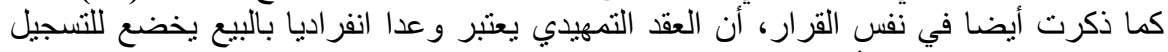

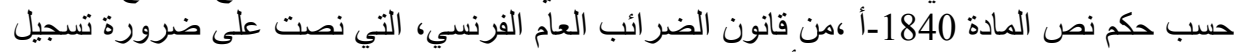

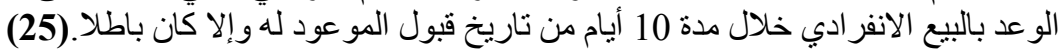

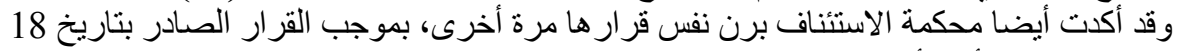

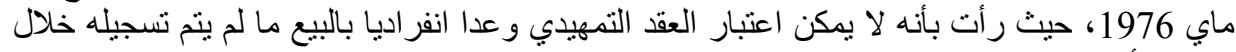

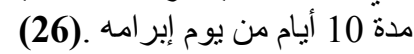

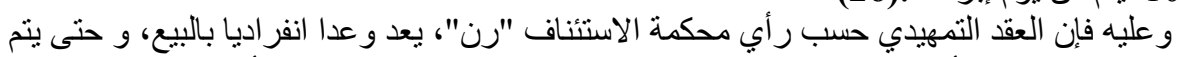

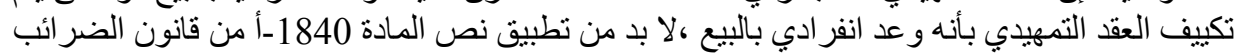

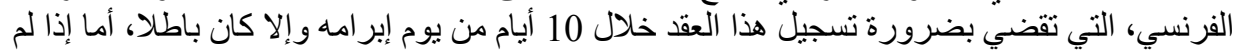

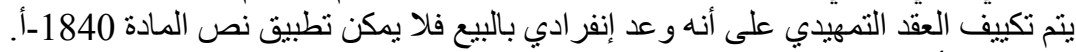

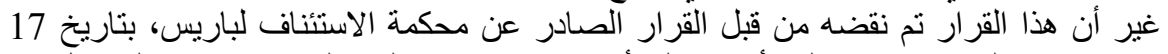

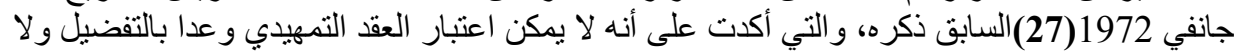

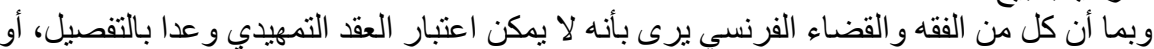

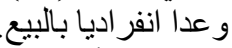

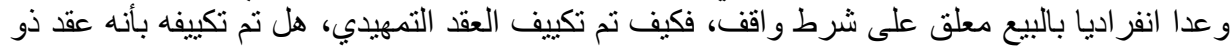
طبيعة مزدوجة، أم أنه عقد ذو طبيعة خاصةٌ هذا ما سوف نعالجه في المرحلة المو الية. 


\section{ثالثا: الاتجاه القائل بأن عقد حفظ الحق عقد ذو طبيعة مزدوجة}

أمام الانتقادات الحادة التي وجهت إلى التكييفات السابقة للعقد التمهيدي، حاول بعض الفقهاء إيجاد التهاد

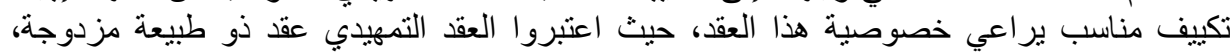
تختلف من حالة إلى أخرى تبعا للوقت الذي أبر أبرم فيه هذا العقد.

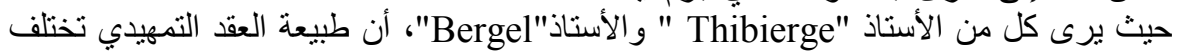

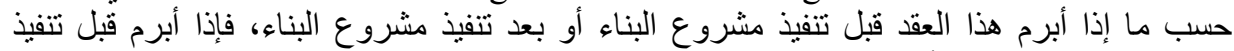

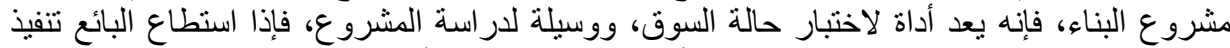

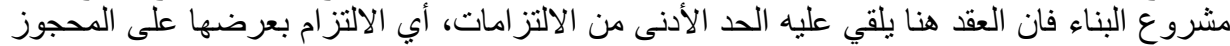

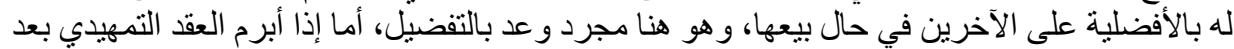

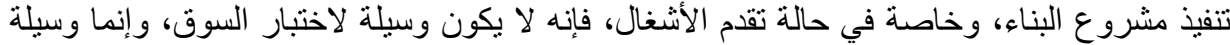

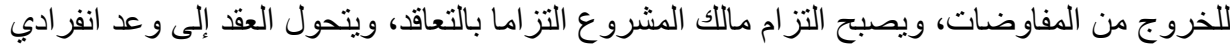

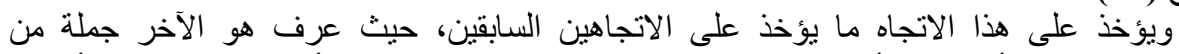

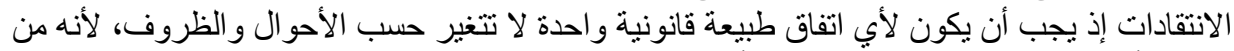

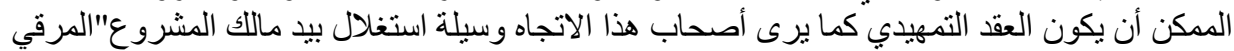

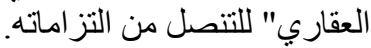

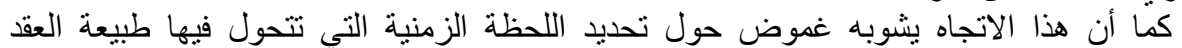

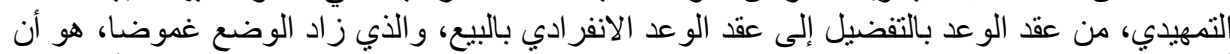

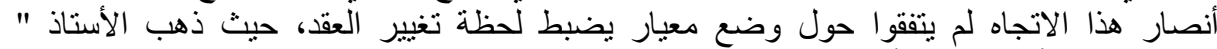
Bergel

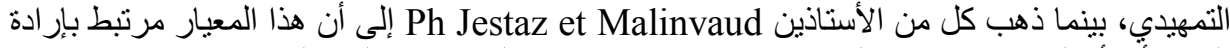

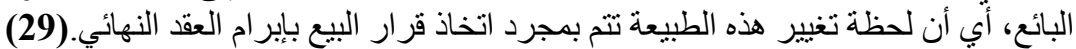

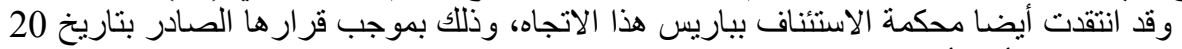

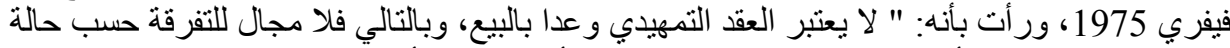

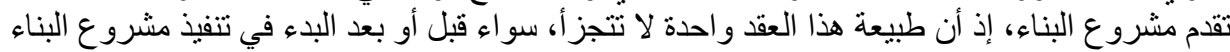

(30)."

\section{رابعا: الاتجاه القائل بأن عقد حفظ الحق عقد ذو طبيعة خاصة}

يتم تكييف عقد ما بأنه عقد ذو طبيعة خاصة، عندما يكون هنالك خلل في التصنيف، أي استحالة التها

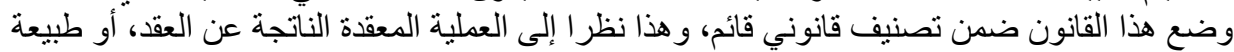

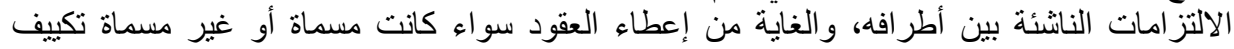

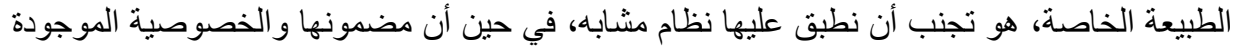

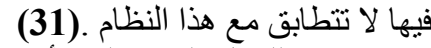

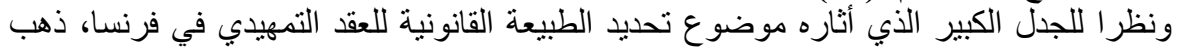

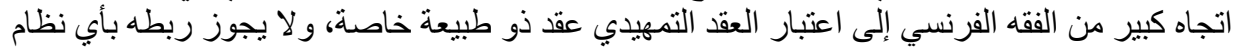




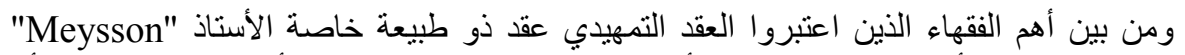

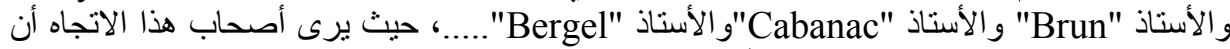

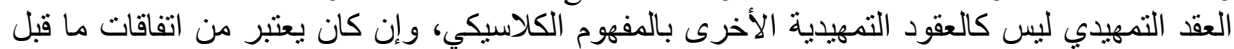

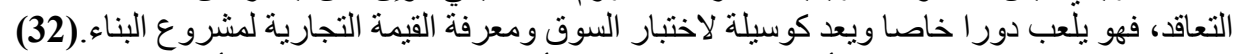

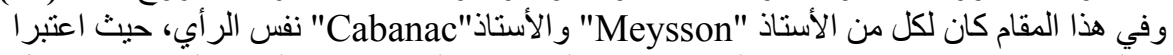

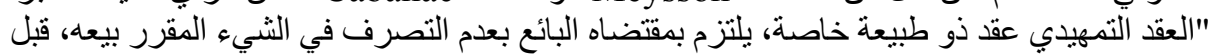

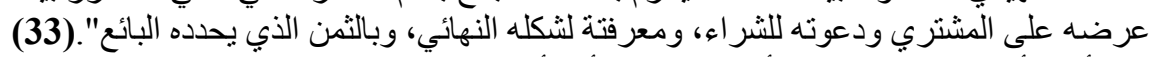

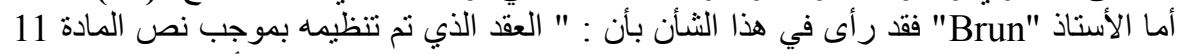

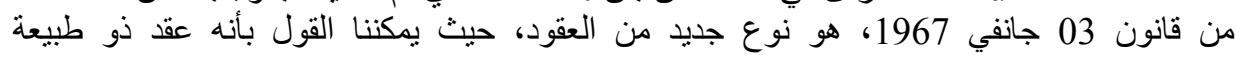

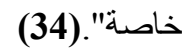

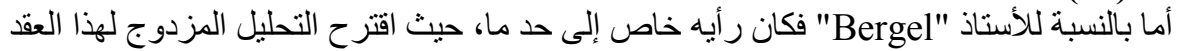

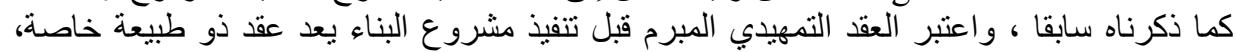

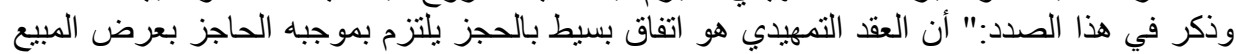

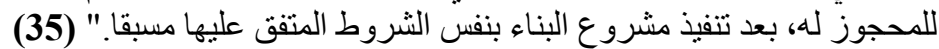

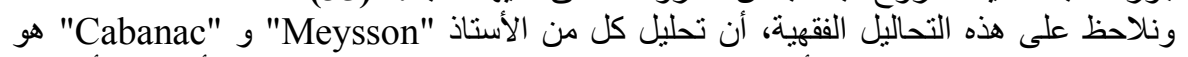

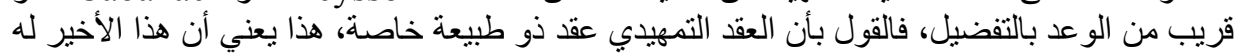

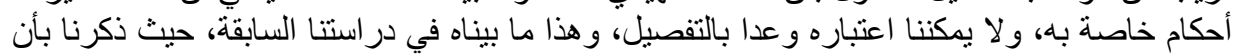

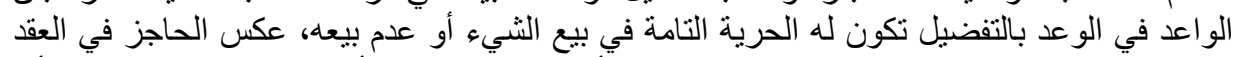

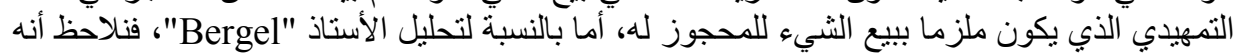

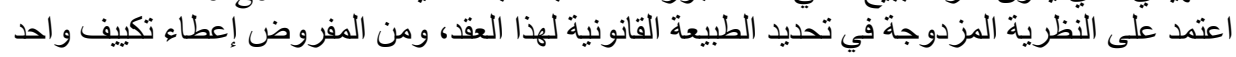

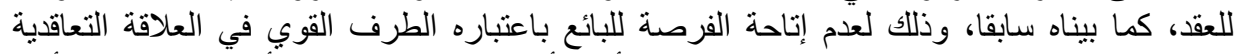

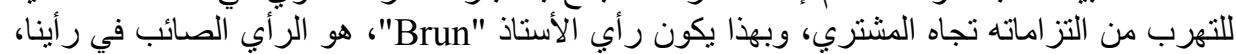

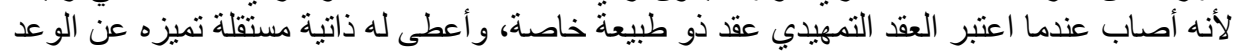
بالتفضيل وعن الو عد الانفر ادي بالييع. وبعد أن تطرقنا إلى رأي الفقه الفرنسي حول الطيع الطبيعة القانونية للعقد التمهيدي، بقي أمامنا معرفة

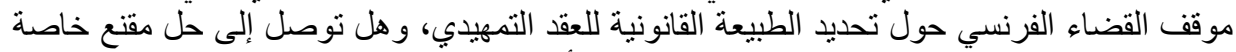

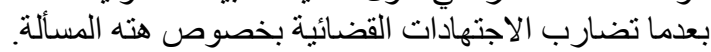

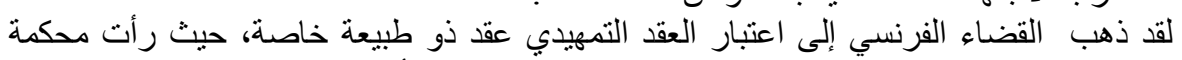

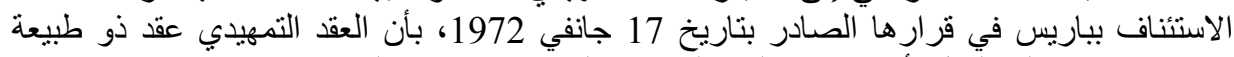

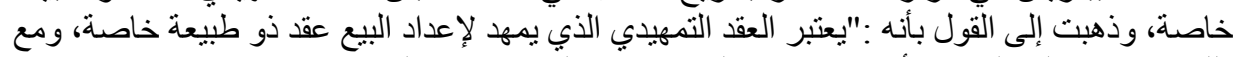

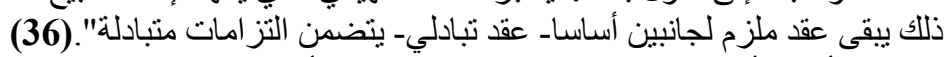

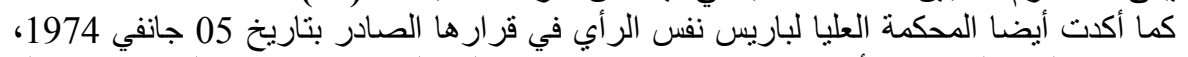

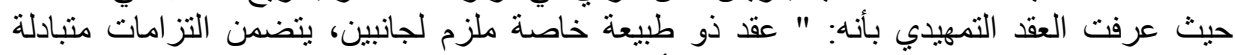

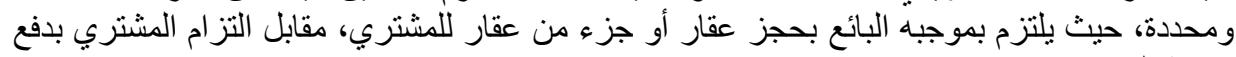

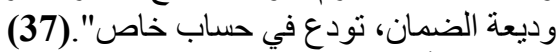

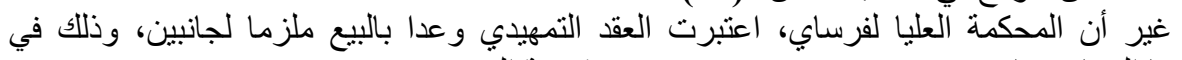

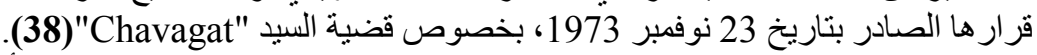

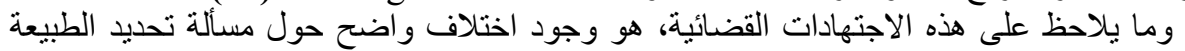
القانونية للعقد التمهيدي، حيث ذهبت كل من محكمة الاستئناف و المحكمة العليا لباريس إلى اعتبار العقد 
التمهيدي عقد ذو طبيعة خاصة ملزم لجانبين ، أما المحكمة العليا لفرساي اعتبرت العقد التمهيدي وعد وقد حسمت محكم لجنين.

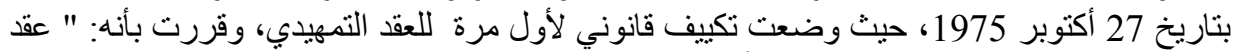

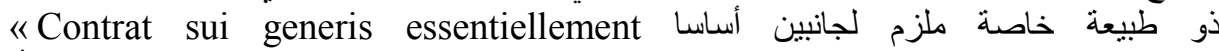

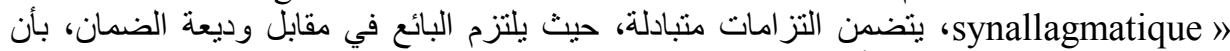

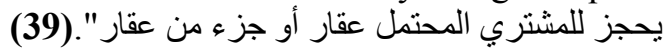

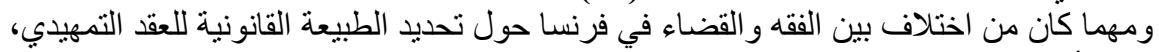

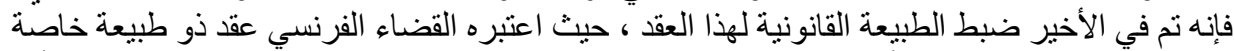

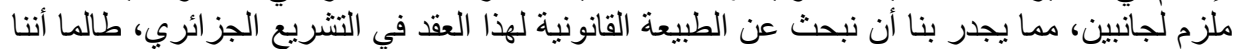

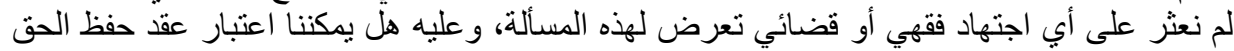

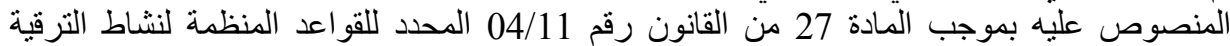

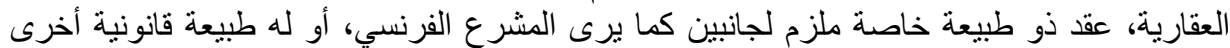
تميزه عن غيره من العقود د.

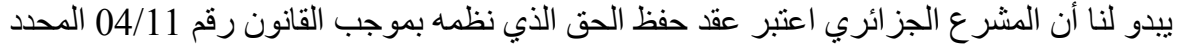

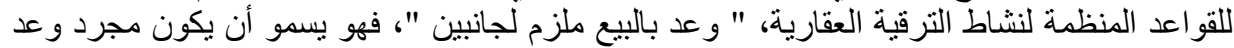

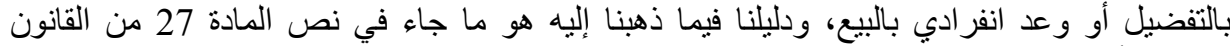

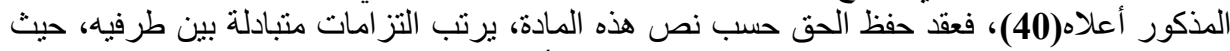

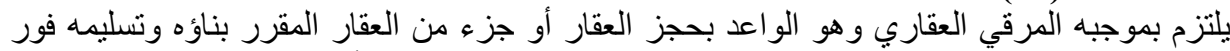

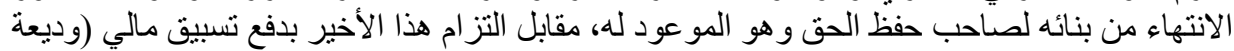

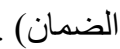

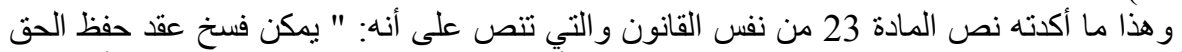

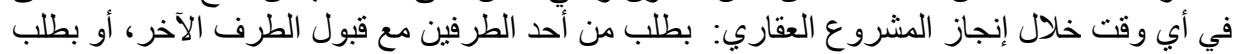

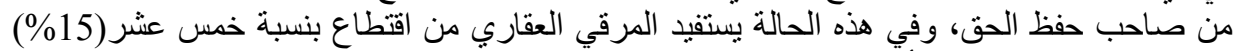

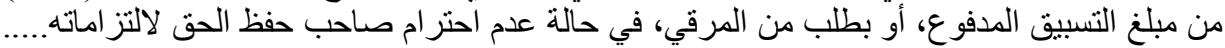

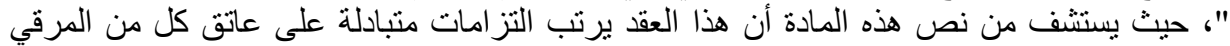

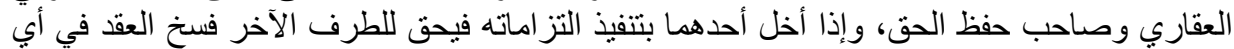
وقت خلال إنجاز مشروع البناء.

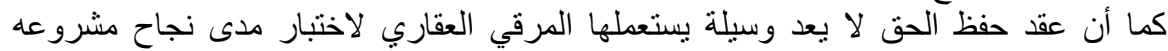

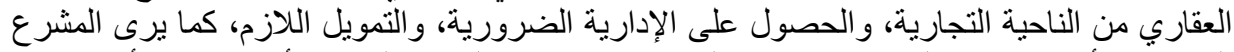

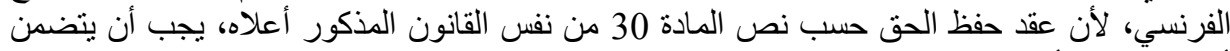

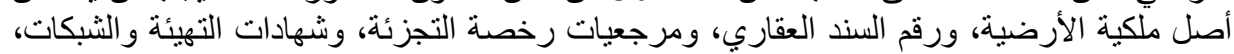

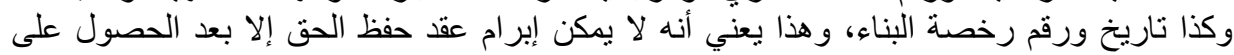
التراخيص اللازمة لمشروع البناء،وبالتالي فإن التزام المرقي العقاري بموجب هذا العادي العقد هو التزام

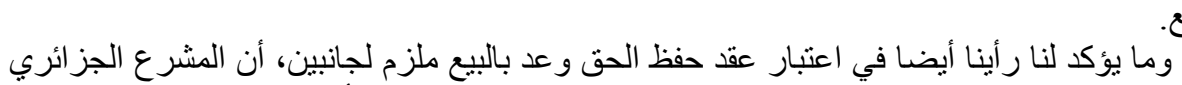

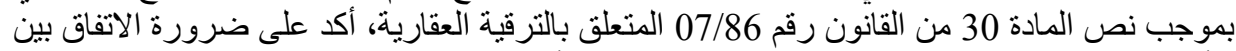

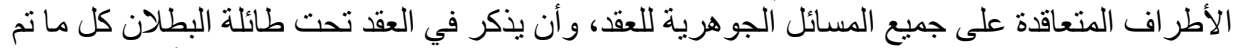

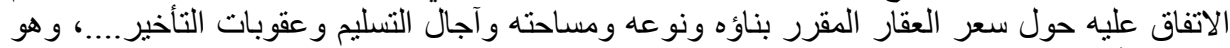

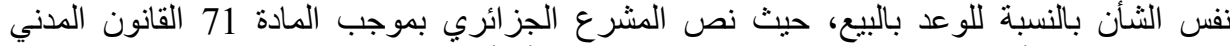

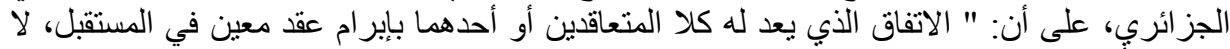

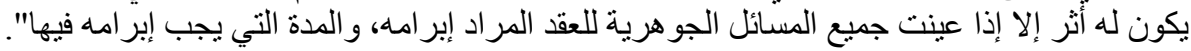


بالإضافة إلى أن المشرع الجزائري أثنار بموجب نص المادة 31 القانون رقم 07/68 المذكور

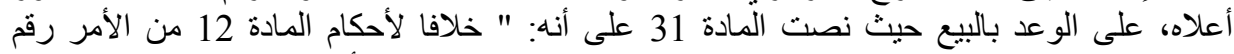

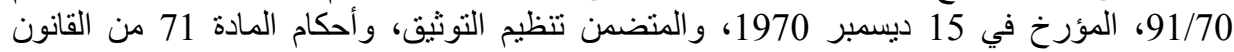

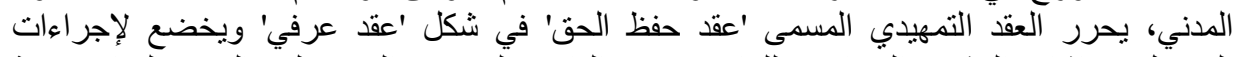

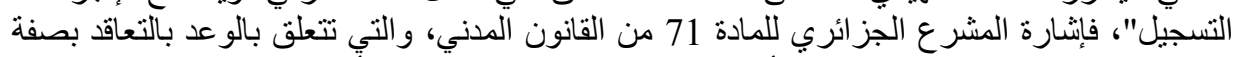

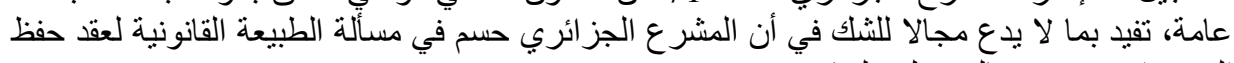

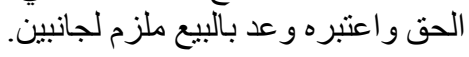

ماتِّة نستخلصه في هذه الدر اسة أن المشرع الفرنسي ورغم تعدد الآراء الفقهية الاجتهادات القضائية

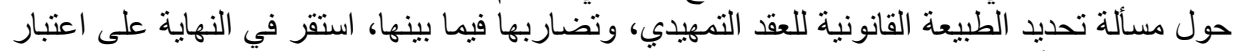

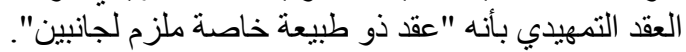

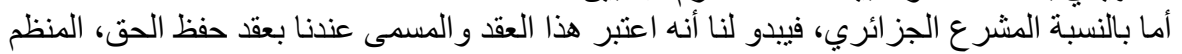

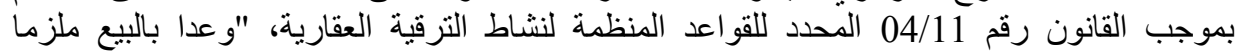

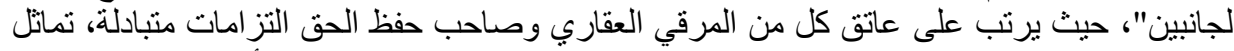

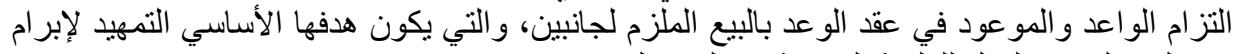
عقد الييع النهائي الناقل للملكية العقارية في المستقبل.

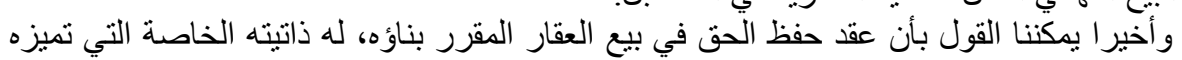

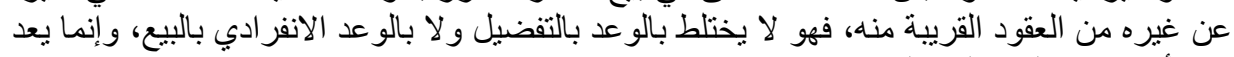
في راينا وعدا بالبيع ملزما لجانبين. 


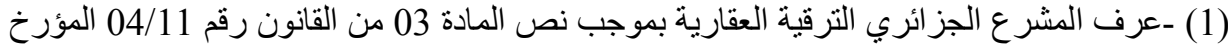

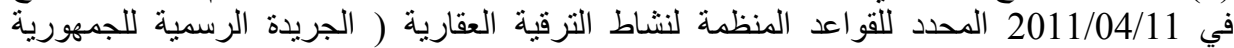

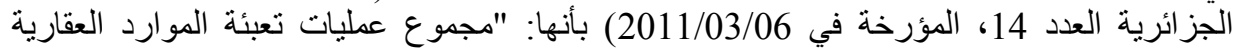
و والمالية وكذا إدارة المشاريع العقاريةّ".

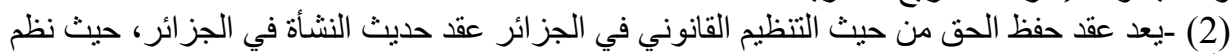

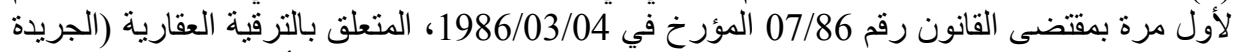

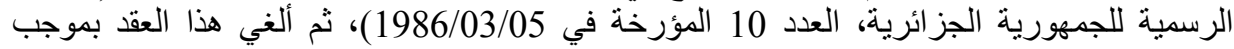

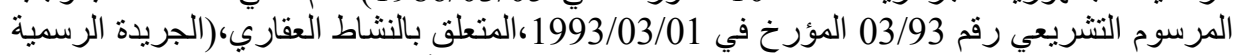

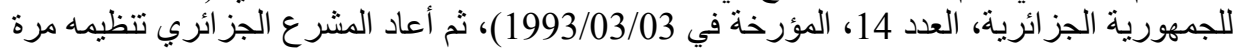

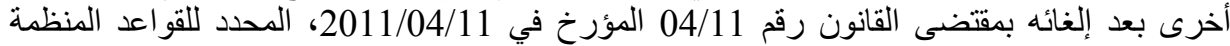
لنشاط الترقية العقارية.

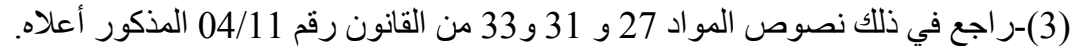

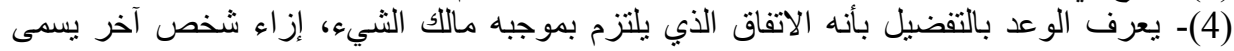

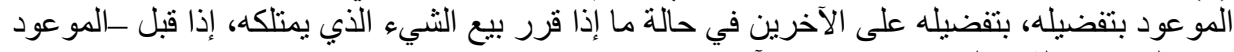
بتفضيله- بدفع الثمن الذي يقدمه شخص آخر.

Theirry Massis ,Le contrat préliminaire dans la vente d'immeuble à construire, thèse, Paris 2, 1979, p 174.

ـ عبد الرزاق أحمد السنهوري، الوسيط في شرح القانون المدني الجديد، العقود التي تقع على الملكية،

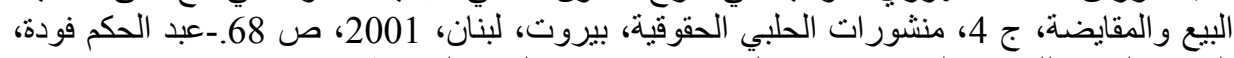

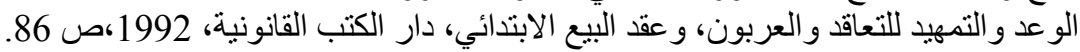
(5)- Saint Alary, La vente d'immeuble à construire et l'obligation de garantie à raison des vices de construction, J-C-P, 1986, N 2146, p 47.

(6)- CL Thibierge, La nouvelle réglementation des ventes d'immeubles à construire, rev.eco.et.dr.imm, n 28, 1967, p 17.

(7)- Therry Massis, thèse précité, p 175,176.

- جميل الثرقاوي، شرح العقود المدنية، البيع والمقايضة، دار النهضة العربية، القاهرة، مصر، 1991،

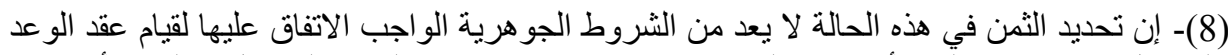

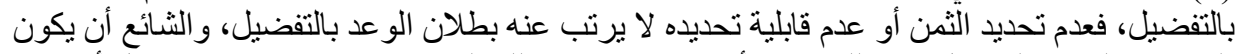

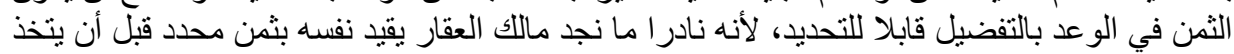

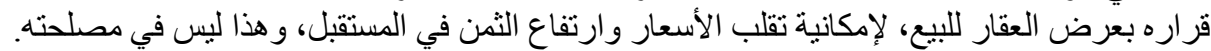

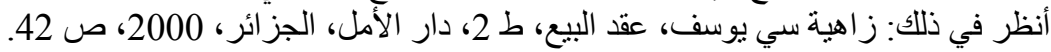
(9) -إبر اهيم عثمان بلآل، تمليك العقار اتل تحت الإنثاء، رسالة دكتور راه، جامعة عين شمس، مصر، (10) 2011، ص اله 49.

(10)- عبد الحفيظ مشماشي، بيع العقار في طور الإنجاز، دراسة على ضوء القانون المغربي و المقارن،

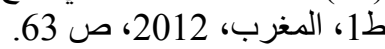

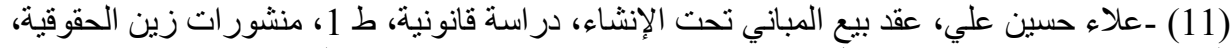

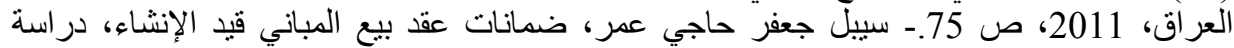

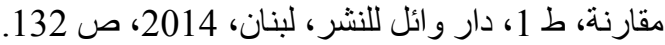

-Voir en ce sens : Therry Massis, thèse précité, p 176 
(12) ـأنظر في ذلك: عيانثي شعبان، عقد بيع العقار بناء على التصاميج، دراسة مقارنة بين القانون

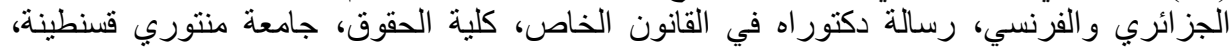

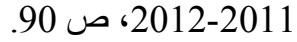

(13)-Therry Massis, thèse précité, p 177,179.

أنظر في ذلك أيضا: - محدد المرسي زهرة، بيع المباني تحت الإنثاء، دراسة مقارنة في القانون

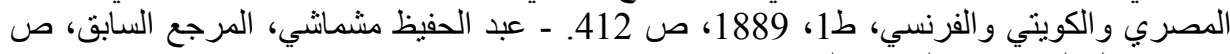

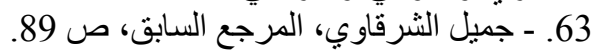

(14)- Cour d'appel de Paris, 17/01/1972, J.C.P, 1972.2.17237, note Meysson et Tirard.

تقول محكمة باريس في هذا الصدد:

" pour certains auteurs, le contrat préliminaire serait un pacte de préférence, la jurisprudence semble bien avoir condamnés cette analyse, en remarquant qu'à la différence du promettant du pacte de préférence, le réservant n' a pas liberté discrétionnaire de ne pas vendre »

(15) ـالوعد الإنفرادي بالبيع هو ذلك العقد الذي بلتزم بمقتضاه صاحب الثنيء بيبعه بثمن معين

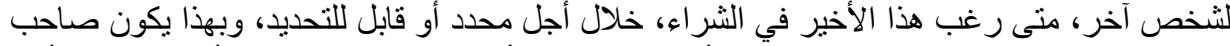

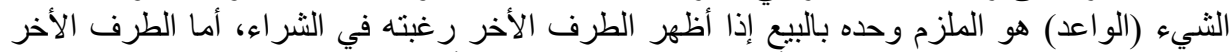

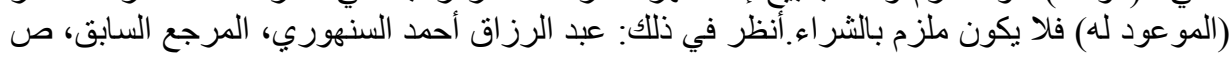

(16)- Frank Steinmetz, les ventes d'immeubles à construire, thèse, Montpelier,

1970, p 316. Therry Massis, thèse précité, p 198,199.

(17)- Frank Steinmetz, thèse précité, p 316.

(18)- François Magnin, la distinction entre contrat de réservation et promesse unilatérale de vente d'immeuble, recueil Dalloz, sommaire commenté, 1993, p 36.

(19)-نص المادة 11 فقرة 4 من القانون رقم 67/03 المؤرخ في 1967/01/03 المتعلق بيع العقار

«.....nulle toute autre promesse d'achat ou vente »

المقرر بناؤهوضمان عيوب البناء :

(20)- Jean Louis Bergel, les contrats préliminaire de réservation dans les ventes :d'immeubles à construire, unité ou dualisme ? ,J.C.P, 1974.1 , N 14, P 2669.

(21)- L'article 11 de la loi du 03 janvier 1967 : «la vente peut être précédée d'un contrat préliminaire par lequel en contrepartie d'un dépôt de garantie effectué à un compte spécial le vendeur s'engage à réserver à un acheteur un immeuble ou une partie d'immeuble »

(22)- Arrêt de cour d'appel commercial Rennes, 18 décembre 1973, Gaz. Pal, 1974.1, P 258. Note M Peisse - D 1974, P 688, note J.C Grosliére.

(23) ـلقد ذهبت محكمة الاستئناف برن إلى اعتبار العقد التمعيدي و عد انفر ادي بالبيع، في قضية الآنسة

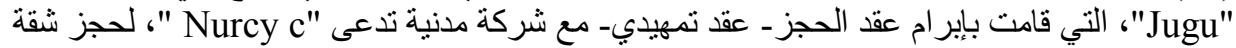

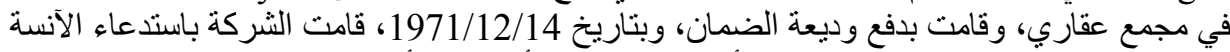

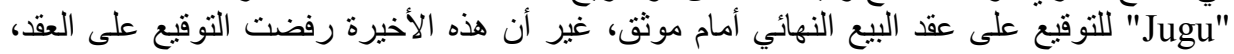


وطلبت من الثركة استرداد وديعة الضمان التي دفعتها، وذللك على أساس أن العقد التمعيدي المبرم

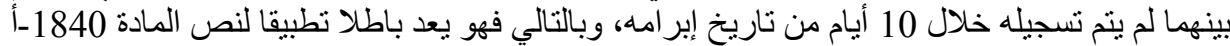
من قانون الضر ائب العام الفرنسي.

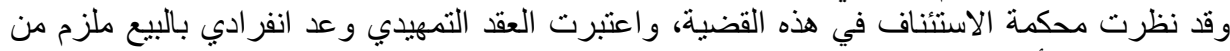

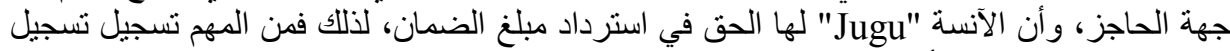
العقد التمهيدي خلال 10 أيام من يوم إبرامه.

Voir en ce sens:- Therry Massis, thèse précité, p 200. - Alain Castel, vente d'immeuble à construire, Etudes et documents de L'.I.E.J. de Rennes, volume 3, N 2, pp 35-36.

(24)- La cour de Rennes confirma cette décision dans les termes suivants : «..Considérant que le contrat préliminaire signé par les parties constitue bien une promesse unilatérale de vente comme le mentionne d'ailleurs le législateur en édictant l'article 11 de la loi du 3 janvier 1967 "est nulle autre promesse d'achat ou de vente "... ».

(25)- « ...considéré que le contrat préliminaire signé par les parties constituait une promesse unilatérale de vente, et comme tel se trouvait soumis aux dispositions de l'article 1840-A du code générale des impôts, qu'ils sont à juste titre déclaré ce contrat nul, comme n'ayant pas été enregistré dans les 10 jours »

(26) -Arrêt de cour d'appel de Rennes, 18/05/1976, J.C.P, 1977.note Maurocq et Stémmer.

Voir en ce sens : Christiane Loyer Larher, vente d'immeuble à construire, Etudes et documents de L'.I.E.J. de Rennes, 1976, volume 7, N 2, pp 13-16. (27)-Cour d'appel de Paris, 17 janvier 1972, précité.

(28)- Therry Massis, thèse précité, p 269 à 300.

$$
\text { (29) -عبد الحفيظ مشماثي، المرجع السابق، ص } 64.18151 .
$$

(30)- Arrêt de cour d'appel de Paris, 20 février 1975,J.C.P, 1975.2.18151, note Bergel.

(31)- Pascal Puig, Contrat spéciaux, Ed, D, 2 ed , 2007, P29.

(32)- Therry Massis, thèse précité, $\mathrm{p} 247$.

(33) -Voir en ce sens : P. Meyssone, Les ventes d'immeuble à construire, J.C.P, 1968, Ed .N.1968.1.2132 ,p 259. - Cabanac, Traité de la construction et de la copropriétaire, T 2, 1970, N 50, P65. Cette analyse est reprise par : Therry Massis, thèse précité, P248.249.

(34)- Ibid, p248.

(35)- Ibid.

(36)- Cour d'appel de Paris, 17 janviers 1972, précité.

La Cour d'appel de Paris confirma cette décision dans les termes suivants : « Considérant toutefois que si ce contrat préliminaire destiné à préparer la vente est un contrat sui generis, il n'en demeure pas moins essentiellement un contrat synallagmatique comportant des obligations réciproques ». (37)- Tribunal de Grande Instance de Paris, 05 janviers 1974, inédit. 
Le Tribunal de Grande Instance dans sa décision en date de 5 janviers 1974, définit le contrat préliminaire comme : «Un contrat synallagmatique sui generis qui comporte des obligations réciproques définies, le vendeur éventuel s'engageant, en contrepartie d'un dépôt de garantie, effectué à un compte spécial, à réserver à l'acheteur éventuel un immeuble ou une partie d'immeuble ».(voir en ce sens : Therry Massis, Thèse précité, P181. (38)- Tribunal de Grande Instance de Versailles, 23 novembre 1973, inédit. لقد ذهبت المحكمة العليا لفرساي في قرارها الصادر بتاريخ 23 نوفمبر 1973، إلى اعتبار العقد

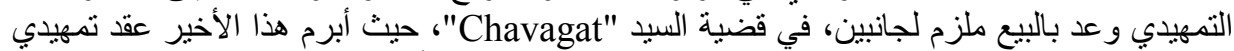

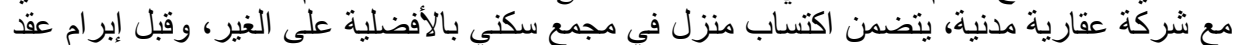

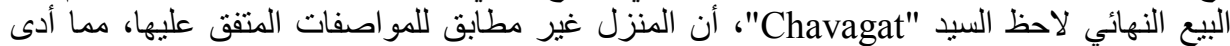

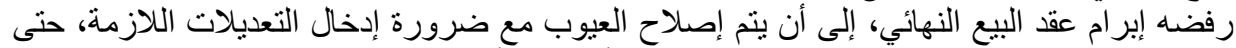

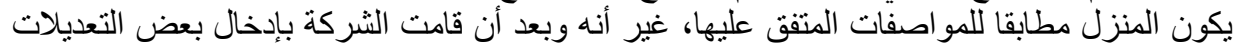

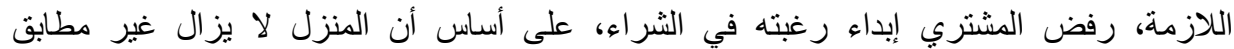

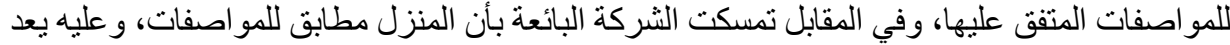

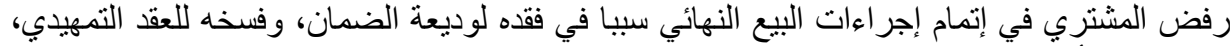

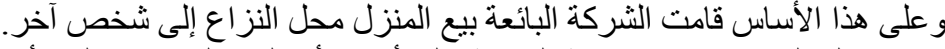

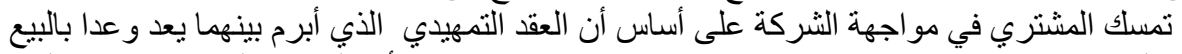

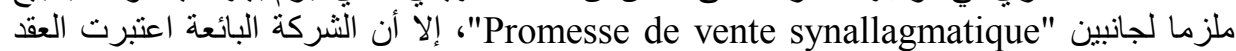
التمهيدي عقد ذو طبيعة خاصنة.

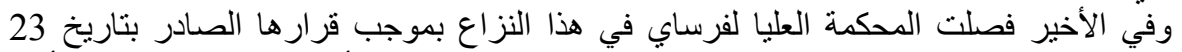

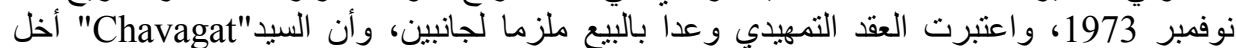

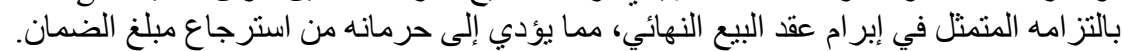

Voir en ce sens : Therry Massis, thèse précité, P 185.186.187.

(39)- cour de Cassation, Civ, 3ch, 27 octobre 1975, et note E. Frank, Gaz. Pal, 1976.1.67.

La cour de cassation confirma cette décision dans les termes suivants : « ....qu'il s'agit d'un contrat sui generis essentiellement synallagmatique, lequel comporte des obligations réciproque, le vendeur s'engageant en contrepartie d'un dépôt de garantie à réserver à l'acheteur éventuel un immeuble ou un partie d'immeuble ».

Voir en ce sens : Therry Massis, thèse précité, p 181.

(40) -عرف المشر هذا التعريف الجزائري عقد حفظ الحق بموجب نص المادة 27 من القانون رقم

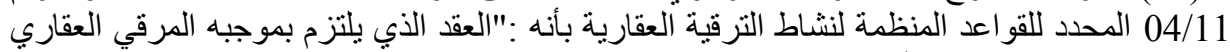

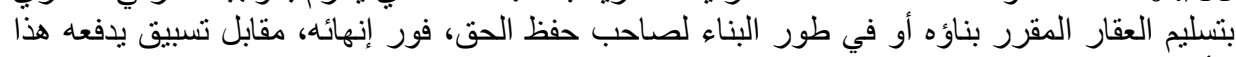

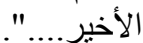

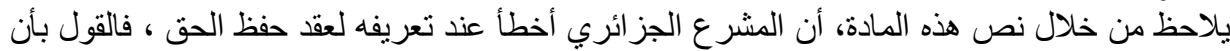

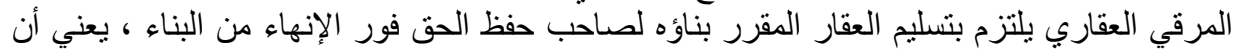




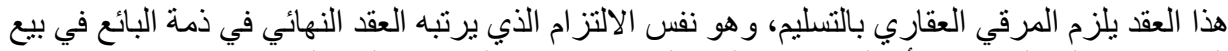

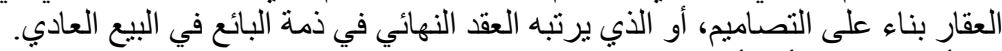

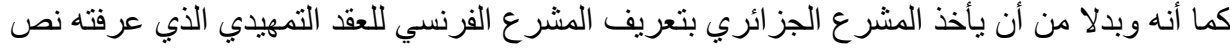

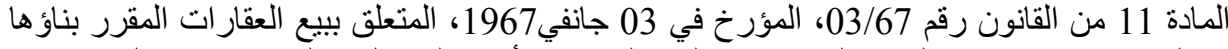

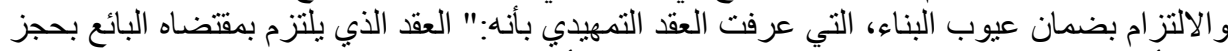

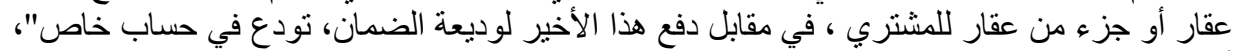

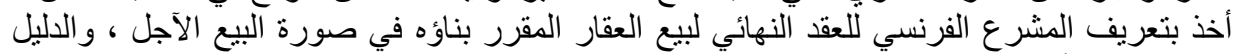

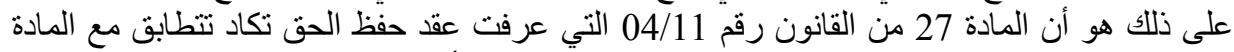

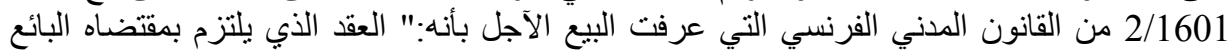

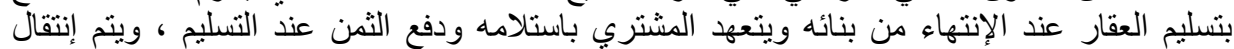

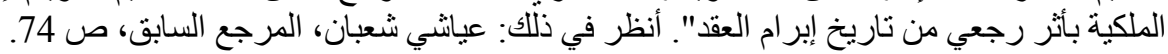

